Editorial

\title{
Acknowledgment to Reviewers of Conservation in 2021
}

\section{Conservation Editorial Office}

MDPI AG, St. Alban-Anlage 66, 4052 Basel, Switzerland

Rigorous peer-reviews are the basis of high-quality academic publishing. Thanks to the great efforts of our reviewers Conservation was able to maintain its standards for the high quality of its published papers. Thanks to the contribution of our reviewers in 2021 the median time to first decision was 13 days and the median time to publication was 33 days. The editors would like to extend their gratitude and recognition to the following reviewers for their precious time and dedication regardless of whether the papers they reviewed were finally published:

Ana Eusébio

Andrea Sujová

Andreia C. M. Rodrigues

Andriy Novikov

Antonija Kulaš

Antonio Cubero-Hernández

Behnaz Safar Ali Najar

Bishnu Hari Poudyal

Blanca R. Lopez

Budi Hadi Narendra

C. Sylvie Campagne

Claudia Canedoli

Cristina Brito

David Valenzuela-Galván

Emanuela Cicinelli

Emiliano Mori

Fabio Cianferoni

Fatmaelzahraa Hussein

Francesco Cerini

Francesco Sica

Francisco Mateos Claros

Franz Krause

George Poinar

Giovanni Bacaro

Giuseppe Brundu

Giuseppe Panarello

Gregorio Vono

Han Van Dobben

Ishwari Singh Bisht

Izabela Krupinska

Jean Yves Heurtebise

Jeffrey C. F. Ho

Jiaying Shi

Jodi L. Sedlock
Jorge L. Ramirez

José Blanco-Salas

Jose Jimenez

José-Francisco Jiménez-Díaz

Ju-Hyoung Lee

Karen S. Oberhauser

Kinga Kostrakiewicz-Gierałt

Krisztián Frank

Krzysztof Młynarczyk

Linas Balčiauskas

Luis Da Costa

María Salinas

Mark C. Belk

Mary Jane Parmentier

Mauricio Hincapié

Michael John Dougherty

Mystera M. Samuelson

Nan-Jay Su

Natalya Ivanova

Nowak Anna

Olga V. Zaharova

Pankaj Kumar Gupta

Parisa Goker

Parsa Arbab

Pawel Wolniewicz

Pedro Mauricio Acosta Castellanos

Phyllis C. Lee

Piero Morseletto

Roberto Amerigo Papini

Roberto Carlucci

Romina Alina Marc

Rositsa Yaneva

Roxana Acosta Gutiérrez

Sabuj Kanti Majumder 
Shankar Adhikari

Yalan Liu

Vasilios Liordos 